\title{
Observation of highly dispersive surface states on $\mathrm{GaN}(0001) 1 \times 1$
}

\author{
Y.-C. Chao, C. B. Stagarescu, J. E. Downes, P. Ryan,* and K. E. Smith ${ }^{\dagger}$ \\ Department of Physics, Boston University, Boston, Massachusetts 02215 \\ D. Hanser, M. D. Bremser, and R. F. Davis \\ Department of Materials Science and Engineering, North Carolina State University, Raleigh, North Carolina 27695
}

(Received 16 February 1999)

\begin{abstract}
The electronic structure of $n$-type, Si-doped, wurtzite $\mathrm{GaN}(0001) 1 \times 1$ surfaces has been studied using synchrotron radiation excited angle-resolved photoemission. The GaN thin films were grown by metal-organic chemical-vapor deposition on SiC. Two previously unobserved surface bands were measured and fully characterized. One of the states is highly nonlocalized, dispersing throughout much of the valence band along the $\bar{\Gamma}-\bar{K}-\bar{M}$ and $\bar{\Gamma}-\bar{M}$ directions of the $1 \times 1$ surface Brillouin zone. The identification of these states as surface bands was confirmed both by their lack of dispersion perpendicular to the surface, and by the sensitivity of the states to hydrogen adsorption. The symmetry properties of the states were determined using the linear polarization of the incident synchrotron radiation. These states are quite distant from the localized nondispersive surface state previously observed on GaN. [S0163-1829(99)50824-2]
\end{abstract}

The physical properties of $\mathrm{GaN}$ and related wide band gap nitride semiconductors are presently under intense study due to their use in optoelectronic and high-temperature semiconductor devices. ${ }^{1-3}$ A fundamental understanding of the electronic structure of these materials is required if they are to achieve their full technological potential. To this end, understanding the electronic properties of clean and adsorbate covered surfaces is of particular importance since it is the first step in understanding fundamental issues of contact formation, chemical reactivity, growth processes, and structural stability. Epitaxial GaN films are commonly prepared by growth on the basal plane of sapphire or Si-terminated $\mathrm{SiC}(0001)$ and this is generally believed to lead to a (0001) growth surface of the $\mathrm{GaN}$ overlayer. ${ }^{4,5}$ Despite numerous studies that monitor the epitaxial process and characterize the resulting morphology of the thin films, the growth front is not yet understood completely. ${ }^{6-12}$ Reconstructions of $1 \times 1,2 \times 2$, and $4 \times 4$ have been reported and demonstrated to correspond to a stable growth front leading to high-quality epitaxial GaN layers. ${ }^{9,13,14}$ Recently, studies based on reflection high-energy electron diffraction concluded that the surface stoichiometry depends on details of the growth parameters such as the substrate temperature, buffer layer, and gallium and nitrogen flux, and even showed a reversible transition at some growth stages by changing these parameters. ${ }^{12}$ There is also much debate concerning the termination and polarization of surfaces of films grown by different methods that are atomically cleaned following removal from the growth chamber. ${ }^{15}$

In the present paper, we report the result of an extensive synchrotron radiation excited angle-resolved photoemission study of $n$-type wurtzite $\mathrm{GaN}(0001) 1 \times 1$ surfaces. The $\mathrm{GaN}$ thin films were grown by metal-organic chemical-vapor deposition (MOCVD) on SiC. Two previously unobserved surface bands were measured and fully characterized. One of the stairs is highly nonlocalized, dispersing throughout much of the valence band along the $\bar{\Gamma}-\bar{K}-\bar{M}$ and $\bar{\Gamma}-\bar{M}$ directions of the $1 \times 1$ surface Brillouin zone. The identification of these states as surface bands was confirmed both by their lack of dispersion perpendicular to the surface, and by the sensitivity of the states to hydrogen adsorption. The symmetry properties of the states were determined using the linear polarization of the incident synchrotron radiation. These states are quite distinct from the localized nondispersive surface state that we observed previously on molecular-beam epitaxy grown $\mathrm{GaN}$ surfaces prepared in an identical manner.

The angle-resolved photoemission (ARP) experiments were performed at the National Synchrotron Light Source (NSLS), on the Boston University/North Carolina State University/NSLS bending magnet beam line U4A, which is equipped with a 6-m toroidal grating monochromator and a custom designed hemispherical electron analyzer. ${ }^{16}$ Typical energy and full angular resolution were $150 \mathrm{meV}$ and $1^{\circ}$, respectively. The base pressure of the ARP chamber was 8 $\times 10^{-11}$ Torr. The $n$-type, Si-doped, wurtzite GaN film was grown on a AlN buffer layer on a $6 \mathrm{H}-\mathrm{SiC}(0001)$ Si-face, on-axis substrate using a low-pressure metal-organic vapor phase epitaxy technique described elsewhere. ${ }^{17}$ Clean surfaces were prepared by repeated cycles of sputtering with $500-\mathrm{eV} \mathrm{N}_{2}$ ions and annealing in ultrahigh vacuum at $850^{\circ} \mathrm{C}$. A very sharp $1 \times 1$ low-energy electron diffraction (LEED) pattern was observed from the surface after cleaning. Auger electron spectroscopy showed a very low surface oxygen level, less than $1 \%$, and no other contamination. (For a comprehensive discussion of the relative merits of various surface cleaning techniques for $\mathrm{GaN}$, see the extensive reviews by Bermudez, Koleske, and Wickenden, ${ }^{15}$ and King et al. ${ }^{10}$ ) Contamination from residual gases in the UHV chamber required that the surface be cleaned every $2 \mathrm{~h}$. Contamination was monitored by the quality of the ARP spectra, which were far more sensitive than AES spectra to the presence of adsorbate. The hydrogen exposures were performed with the GaN surface facing a hot, well outgassed W filament, in order to chemisorb atomic hydrogen. Nominal hydrogen exposures of typically $7000-10000 \mathrm{~L}(1 \mathrm{~L}=1$ $\times 10^{-6}$ Torr s) were required to completely remove the sur- 


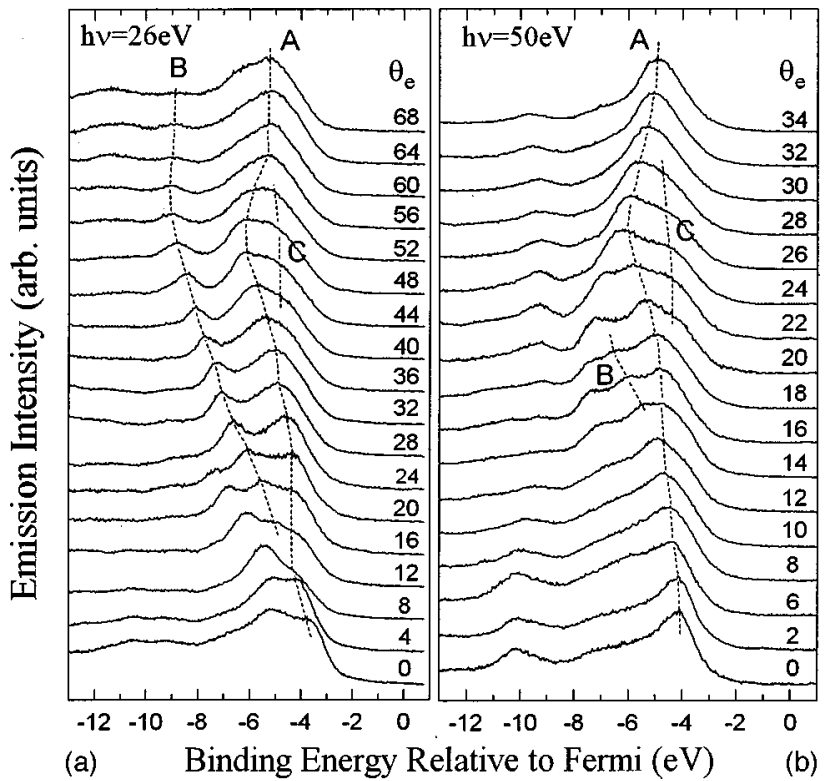

FIG. 1. ARP spectra recorded from the clean $\mathrm{GaN}(0001) 1 \times 1$ surface with photon energies of (a) $26 \mathrm{eV}$ and (b) $50 \mathrm{eV}$ showing emission from states along the $\bar{\Gamma}-\bar{K}-\bar{M}$ direction. The angle of incidence $\left(\theta_{i}\right)$ of the light was $45^{\circ}$.

face states. Since ARP does not provide unambiguous direct information on the termination or polarization of these samples, the film surface references as either (0001) or $(0001)$, with the former used here for convenience. ${ }^{15}$

Figure 1(a) presents ARP spectra from clean $\mathrm{GaN}(0001) 1 \times 1$ where the detector angle was varied to measure emission from states lying along the $\bar{\Gamma}-\bar{K}-\bar{M}$ direction in the surface Brillouin zone. The photon energy used to excite the spectra in Fig. 1(a) was $26 \mathrm{eV}$. Three features in the spectra, labeled $A, B$, and $C$, can be identified as emission from surface states. All other features in the spectra originate in emission from bulk states, and correspond to bulk states observed in an earlier ARP study of wurtzite GaN. ${ }^{6}$ The same three surface related features can be seen in the spectra of Fig. 1(b), where here the incident photon energy $h \nu=50$ $\mathrm{eV}$. State $A$ lies near the valence-band maximum (VBM) at the zone center $\left(\bar{\Gamma}\right.$ point, normal emission, $\left.\theta_{e}=0^{\circ}\right)$, and shows only slight dispersion until far from $\bar{\Gamma}$ when it disperses downward by $\sim 1.5 \mathrm{eV}$ (around the $\bar{K}$ point, as will be shown below). In contrast, state $B$ disperses rapidly away from the $\bar{\Gamma}$ point, extending to the bottom of the valence band at $\bar{K}$.

Figure 2 presents equivalent sets of spectra to those of Fig. 1, except that the detector and film are oriented such that emission from states along the $\bar{\Gamma}-\bar{M}$ direction is being measured. In this azimuth, emission features $A$ and $B$ were clearly observed when $h \nu=26 \mathrm{eV}$, but at $h \nu=50 \mathrm{eV}$ emission feature $B$ was not visible due to stronger emission from an overlapping bulk state. As with emission from states along $\bar{\Gamma}-\bar{K}-\bar{M}$, feature $A$ exhibits very slight dispersion, while feature $B$ exhibits strong dispersion towards the bottom of the valence band away from the zone center.

Emission feature $A$ is highly sensitive to contamination from background gases, and the intensity of this feature is used as a very sensitive indicator of the cleanliness of the

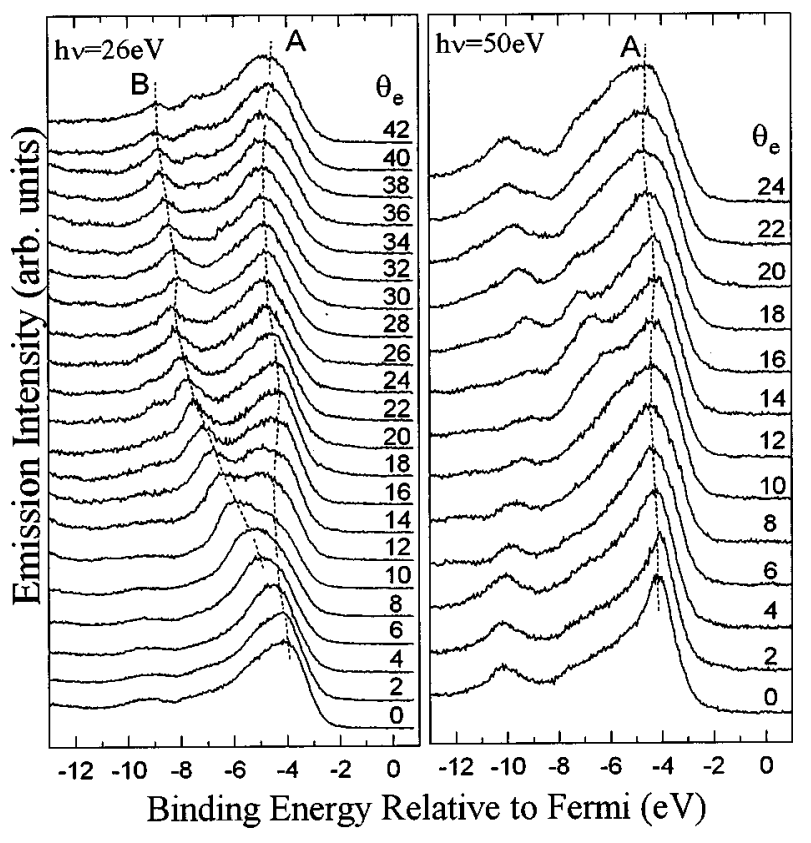

FIG. 2. Same as for Fig. 1, but for states along the $\bar{\Gamma}-\bar{M}$ direction.

surface. Feature $B$ is not as sensitive to background gas contamination, but is a sensitive indicator to the emission of a good $1 \times 1$ reconstruction, as will be discussed below. A weak surface sensitive emission feature (labeled $C$ in Fig. 1) was also observed, appearing near the VBM close to the $\bar{K}$ point. The surface sensitivity of these features is illustrated by the spectra of Fig. 3. The top pair of spectra compare emission taken from two different surfaces: spectrum $(a)$ was taken from a surface with very sharp LEED $1 \times 1$ spots, while

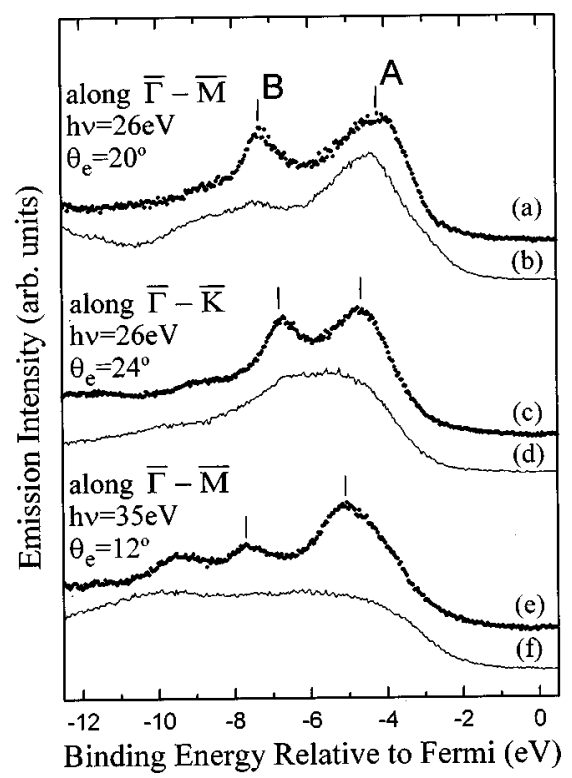

FIG. 3. ARP spectra recorded with different photon energies and azimuthal directions. $\theta_{i}=45^{\circ}$. Spectra $(a),(c)$, and $(e)$ were recorded from the clean $\mathrm{GaN}(0001) 1 \times 1$ surface. Spectrum $(b)$ was recorded from a clean surface with streaky $1 \times 1$ LEED pattern, while spectra $(d)$ and $(f)$ were recorded after saturating the surface with hydrogen. See text. 


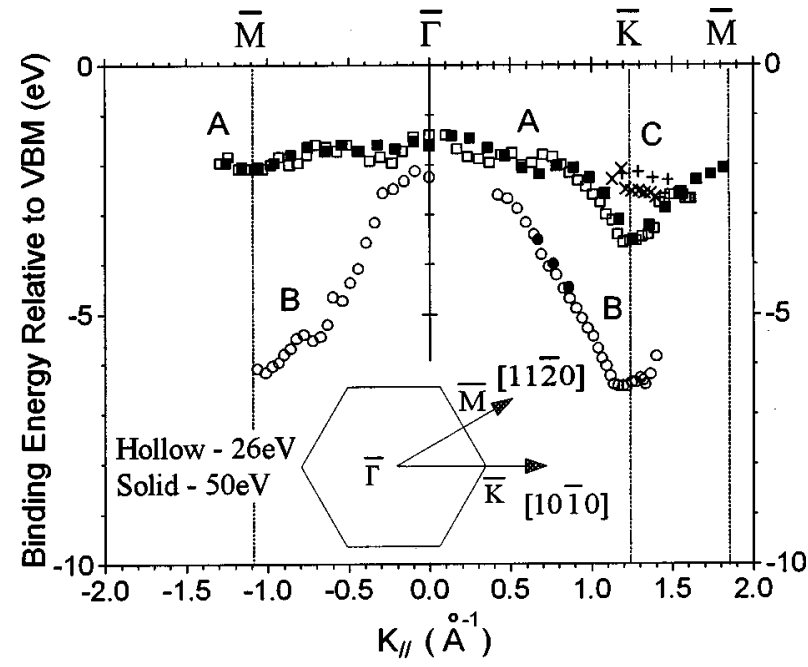

FIG. 4. Measured two-dimensional band structure for the clean $\mathrm{GaN}(0001) 1 \times 1$ surface in the $1 \times 1$ surface Brillouin zone. The recording geometry is indicated in the inset.

spectrum $(b)$ was taken from a surface with streaky LEED $1 \times 1$ spots. Both spectra were recorded immediately following cleaning. As is clear in the figure, feature $A$ is visible in both spectra while feature $B$ appears only weakly in spectrum $(b)$, indicating the sensitivity of this feature to the degree of order of the surface layer. Spectra $(c)$ through $(f)$ in Fig. 3 illustrate the difference between the clean and hydrogen saturated surfaces. Spectra $(c)$ and $(e)$ were recorded from the clean surface while $(d)$ and $(f)$ were recorded from the surfaces exposed to $\sim 10000 \mathrm{~L}$ hydrogen. It is clear that features $A$ and $B$ on the clean surface are completely removed by the hydrogen chemisorption, and that no hydrogen-induced emission features were observed. This is consistent with our LEED measurements: we observe diffuse $1 \times 1$ spots with higher background scattering for the hydrogen saturated surfaces, but no reconstruction.

Assuming a free electron final state, we can use the data of Figs. 2 and 3 to generate the measured surface band dispersion as shown in Fig. 4. ${ }^{18,19}$ Earlier inverse photoemission experiments have shown that a free-electron final state is a good approximation for photon energies above $25 \mathrm{eV} .^{20}$ There are few published calculations of the surface band structure of $\mathrm{GaN}(0001) 1 \times 1$ to which we can compare our data. A surface state has been reported for a 3/4 monolayer of hydrogen on $\mathrm{GaN}(0001)$ by Rapcewicz, Buongiorno Nardelli, and Bernholc, ${ }^{11}$ but this state does not correspond to our measured dispersion.

The exact origins of the observed surface states are difficult to determine due to uncertainties in the structure, termination, and stoichiometry of the surface. However, the characteristics of these surface bands give some important clues. The most notable aspect of the surface state corresponding to emission feature $A$ is the fact that it is highly sensitive to surface contamination. It also shows very little dispersion throughout the $1 \times 1 \mathrm{SBZ}$ until it approaches the $\bar{K}$ point, where a downward dispersion of $\sim 1.5 \mathrm{eV}$ is observed. The lack of dispersion indicates that state $A$ is quite localized, probably corresponding to the dangling-bond surface state observed in earlier studies. The reactivity of this state is con-

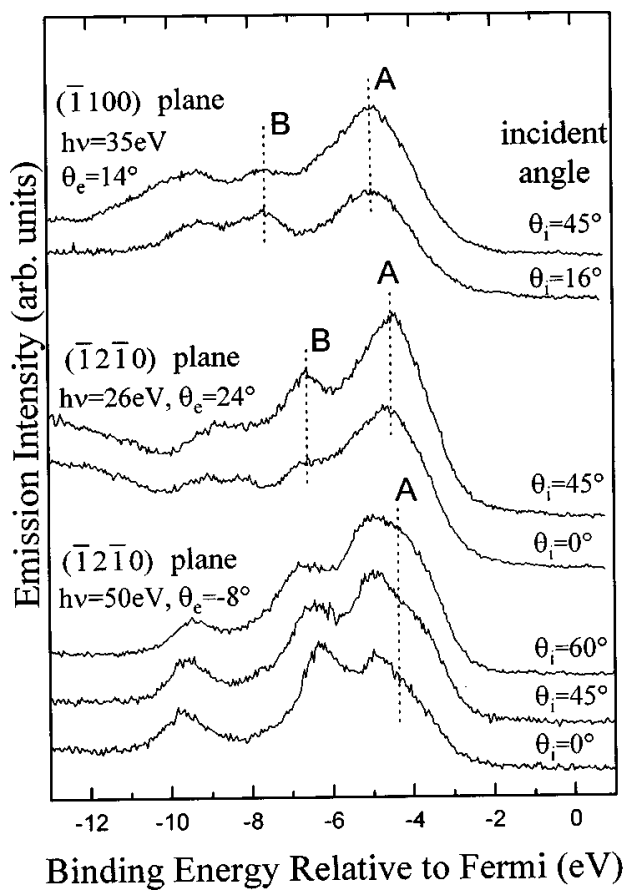

FIG. 5. ARP spectra recorded from the clean $\mathrm{GaN}(0001) 1 \times 1$ surface with different photon energies and azimuthal directions. $\theta_{i}$ $=45^{\circ}$. The details of the symmetry properties of the $A$ and $B$ states are discussed in the text.

sistent with this assignment. The band corresponding to emission feature $B$ shows a strong downward dispersion when moving away from the $\bar{\Gamma}$ point. As noted earlier, this state is less sensitive than feature $A$ to background gas contamination and is well resolved only when the surface exhibits a sharp $1 \times 1$ LEED pattern. A plausible assignment is that this state is a back-bonding state, which generally display a high degree of dispersion.

Further information on these surface states can be obtained by taking advantage of the linearly polarized nature of the synchrotron radiation used to excite the spectra. The symmetry properties of the states can be determined by orienting the synchrotron radiation relative to a high symmetry direction containing a mirror plane of the surface, and applying conventional selection rules. The results for emission features $A$ and $B$ are shown in Fig. 5. Three sets of spectra are presented with different recording geometries. The uppermost pair of the spectra were recorded with the detector along the $\bar{\Gamma}-\bar{M}$ direction, i.e., $[11 \overline{2} 0]$, and the polarization vector in the plane defined by the [0001] direction and the emission direction, which contained [1120]. The remaining spectra were recorded along the $\bar{\Gamma}-\bar{K}-\bar{M}$ direction and the polarization vector in the $(\overline{12} \overline{1} 0)$ plane. According to mirror plane selection rules, the initial states must have a definite even or odd parity with respect to the mirror planes. ${ }^{21}$ The requirement of even final states for electrons emitted in the mirror plane implies that only even initial states will be observed with the above recording geometry. Thus, both the $A$ and $B$ states are even with respect to the $(\overline{1} 100)$ and $(\overline{1} 2 \overline{1} 0)$ mirror planes. By changing the angle of incidence of the synchrotron radiation, it is clear that the intensity of feature $A$ increases with increasing incident angles with respect to the (1100) mirror plane, while the intensity of both the $A$ and 
$B$ features increases with respect to the $(\overline{12} \overline{1} 0)$ mirror plane. The increase of the emission intensities with increasing angle of incidence suggests a strong dependence on the $z$ component of the electric-field vector of the synchrotron radiation, thus indicating a strong $p_{z}$ character with respect to the corresponding mirror planes. However, a full calculation of the surface electronic structure is required to test the origin of these states further.

Finally, the results of this study can be compared directly to our earlier study of the surface electronic structure of GaN. ${ }^{8}$ The wurtzite GaN films used in that study was grown by molecular beam epitaxy (MBE), and although the films were cleaned in an identical manner, the surface electronic structure is quite different. No back-bonding dispersive states were observed at all in the earlier study. A danglingbond state was observed on the surface of the MBE-grown films, but it did not show significant dispersion about $\bar{K}$. It is likely that this localized state is related to surface defects. The different surface electronic structure between the MOCVD and MBE materials cleaned in an identical fashion is probably not related to the growth mode, but rather to differences in surface termination. It should also be noted in this context that although both types of surfaces gave $1 \times 1$ LEED patterns, the patterns from the MOCVD surfaces were of higher quality, with sharper smaller spots and a lower background than we were able to achieve in the earlier study. This is consistent with the sensitivity of the back-bonding state (feature $B$ ) to the surface ordering observed in the present study.

To summarize, the surface electronic structure of a wellordered wurtzite $\mathrm{GaN}(0001) 1 \times 1$ surface has been studied by polarization-dependent angle-resolved photoelectron spectroscopy using synchrotron radiation. Two distinct surface bands are observed. The identification of these surface states was confirmed by the photon energy independence of their dispersion, as well as their sensitivity to hydrogen adsorption. One state $(A)$, near the valence-band maximum, was found to be weakly dispersive and very sensitive to hydrogen adsorption, suggesting it is a dangling-bond state. The second observed state $(B)$ displayed strong dispersion throughout the two-dimensional $1 \times 1$ surface Brillouin zone and was found to depend strongly on the quality of the $1 \times 1$ reconstruction. Both states are of even symmetry with respect to the (1210) and (1100) mirror planes. Moreover, both show a strong dependence on the $z$ component of the electric-field vector of the synchrotron radiation with respect to the $(\overline{1} 2 \overline{1} 0)$ mirror plane indicating a strong $p_{z}$ character.

This work was supported in part by the National Science Foundation under Grant No. DMR-95-04948. Experiments were performed at the NSLS which is supported by the U.S. Department of Energy, Division of Materials and Chemical Sciences. We gratefully acknowledge numerous helpful discussions with V. M. Bermudez.
*Permanent address: School of Physical Sciences, Dublin City University, Dublin 9, Ireland.

†Author to whom correspondence should be addressed. Electronic address: ksmith@bu.edu

${ }^{1}$ S. Strite and H. Morkoç, J. Vac. Sci. Technol. B 10, 1237 (1992).

${ }^{2}$ Diamond, Silicon Carbide and Nitride Wide Bandgap Semiconductors, edited by C. H. Carter, Jr., MRS Symposia Proceedings No. 339 (Materials Research Society, Pittsburgh, 1994).

${ }^{3}$ III-V Nitrides, edited by F. A. Ponce, T. D. Moustakas, I. Akasaki, and B. A. Monemar, MRS Symposia Proceedings No. 449 (Materials Research Society, Pittsburgh, 1997).

${ }^{4}$ F. A. Ponce, D. P. Bour, W. T. Young, M. Saunders, and J. W. Steeds, Appl. Phys. Lett. 69, 337 (1996).

${ }^{5}$ S. Y. Ren and J. D. Dow, Appl. Phys. Lett. 69, 251 (1996).

${ }^{6}$ V. M. Bermudez, J. Appl. Phys. 80, 1190 (1996).

${ }^{7}$ J. Fritsch, O. F. Sankey, K. E. Schmidt, and J. B. Page, Phys. Rev. B 57, 15360 (1998).

${ }^{8}$ S. S. Dhesi, C. B. Stagarescu, K. E. Smith, D. Doppalapudi, R. Singh, and T. D. Moustakas, Phys. Rev. B 56, 10271 (1997).

${ }^{9}$ A. R. Smith, V. Ramachandran, R. M. Feenstra, D. W. Greve, M. S. Shin, M. Skowronski, J. Neugebauer, and J. E. Northrup, J. Vac. Sci. Technol. A 16, 1641 (1997).

${ }^{10}$ S. W. King, J. P. Barnak, M. D. Bremser, K. M. Tracy, C. Ron- ning, R. F. Davis, and R. J. Nemanich, J. Appl. Phys. 84, 5248 (1998).

${ }^{11}$ K. Rapcewicz, M. Buongiorno Nardelli, and J. Bernholc, Phys. Rev. B 56, R12 725 (1997).

${ }^{12}$ P. Hacke, G. Feuillet, H. Okumura, and S. Yoshida, Appl. Phys. Lett. 69, 2507 (1996).

${ }^{13}$ A. R. Smith, R. M. Feenstra, D. W. Greve, J. Neugebauer, and J. E. Northrup, Phys. Rev. Lett. 79, 3934 (1997).

${ }^{14}$ A. R. Smith, R. M. Feenstra, D. W. Greve, M. S. Shin, M. Skowronski, J. Neugebauer, and J. E. Northrup, Appl. Phys. Lett. 72, 2114 (1998).

${ }^{15}$ V. M. Bermudez, D. D. Koleske, and A. E. Wickenden, Appl. Surf. Sci. 126, 69 (1998).

${ }^{16}$ S. D. Kevan, Rev. Sci. Instrum. 54, 1441 (1983).

${ }^{17}$ T. W. Weeks, Jr., M. D. Bremser, K. S. Ailey, E. Carlson, W. G. Perry, and R. F. Davis, Appl. Phys. Lett. 67, 401 (1995).

${ }^{18}$ S. D. Kevan, Angle Resolved Photoemission (Elsevier, Amsterdam, 1991).

${ }^{19}$ K. E. Smith and S. D. Kevan, Prog. Solid State Chem. 21, 49 (1991).

${ }^{20}$ T. Valla, P. D. Johnson, S. S. Dhesi, K. E. Smith, D. Doppalapudi, T. D. Moustakas, and E. L. Shirley, Phys. Rev. B 59, 5003 (1999).

${ }^{21}$ J. Hermanson, Solid State Commun. 22, 19 (1977). 